\title{
Comparison of Near Add by Raf Rule and Age-Based Addition Method
}

\author{
Marian Ahmed ${ }^{1}$ and Ayesha Mushtaq ${ }^{2 *}$ \\ ${ }^{1}$ Optometry, The Doctor Optometry, Pakistan \\ ${ }^{2}$ Allama Iqbal Open University Islambad, AIOU, Pakistan \\ *Corresponding Author: Ayesha Mushtaq, Allama Iqbal Open University Islambad, AIOU, Pakistan.
}

Received: July 01, 2019; Published: September 24, 2019

DOI:10.31080/ASNH.2019.03.0461

\begin{abstract}
Purpose: Use of plus lenses compensate for the reduction in amplitude range caused due to presbyopia. It brings the near point of accommodation of presbyopia patient to a distance which is suitable for performing near tasks. The basic aim of this study was to compare two different methods of prescribing near add in presbyopia patients. The research was carried out with 40 subjects with mean age 61.0(range 40-70 years). Two different methods were used to determine near add, including the amplitude of accommodation by RAF ruler and age based plus lenses method. The add power obtained by both procedures was then refined to reach the final prescription. The results obtained by both procedures showed a notable difference in near addition values. The mean near add values in case of age based method were higher as compared to those with AA by RAF rule method. Age-based method overestimated reading addition relative to other method. Mean near addition obtained by RAF rule method and age-based method were 0.86 and 1.51 , respectively. The results were highly significant with $p$ value of $(\mathrm{p}=0.000)$.
\end{abstract}

\begin{tabular}{|l|c|c|c|c|c|}
\hline & Sum of Squares & df & Mean Square & F & Sig. \\
\hline Between Groups & 8.613 & 1 & 8.613 & 27.800 & .000 \\
\hline Within Groups & 24.167 & 78 & .310 & & \\
\hline Total & 32.780 & 79 & & & \\
\hline \multicolumn{7}{|c|}{ Table }
\end{tabular}

It was concluded that both these methods provided results which showed a marked difference. The results obtained by ageexpected method were nearly close to the final addition. Therefore, the age based method is recommended as it is least time consuming and the probability of error is quite low. Moreover, it gave results which correlated best with final prescription of patient. It is recommended that if possible, the near add should be adjusted according to the particular needs of the patient because the near work demands varies from individual to individual.

Keywords: RAF Ruler; Presbyopia; Accomodation; Amplitude of Accomodation

\section{Introduction}

Presbyopia is not an error of refraction but a condition of physiological insufficiency of accommodation due to reduced amplitude, leading to progressive fall in near vision beginning at 40-45 years of age. The natural crystalline lens of eye is second most refracting surface of eye due to which the light must pass through it to fall on retina. In order to clearly see an image the rays entering in eye should be directly focus on retina. Accommodation is a mechanism in which the crystalline lens of eye changes its shape to focus from distant to near objects. In people below the age of 40 the eye automatically accommodate in order to view close object. After the age of 40 the natural crystalline lens becomes stiff which make it difficult to change the focus according to distance (Khurana A.K, 2013).
The crystalline lens of eye is mainly originated from ectoderm layer therefore its growth continues throughout the life. New fibers are continuously being formed at the equator which is responsible for its growth. Due to this expansion there is a proportional decrease in ciliary muscle length accompanied by a decrease in the strength of ciliary muscles. The formation of new fibers results into gradual thickening and reduced elasticity of natural crystalline lens. Due to sclerosis of lens substance the lens becomes harder and less elastic. The normal reading distance up till the age of 40 years is $25 \mathrm{~cm}$. After the age of 40 , near point of accommodation (NPA) recedes beyond the normal reading range. The initiation of presbyopia depends on a number of different factors such as refractive errors, geographical distribution, profession, systemic diseases, and use of anti-allergic drugs (Wilson D.J, 2019). 
Its now globally recognized that presbyopia is type of refractive error which needs to be managed properly. A good near vision is important to carry out daily life activities including reading, writing, computer work and other vocational jobs. Presbyopic patients present with complain of headache, eyestrain and they usually hold objects further away from eyes to focus clearly. The objects may be in focus but they are too small to be recognized. The common symptoms of presbyopia include blurred near vision, eye ache after a short period of near work and headache. The common risk factor includes uncorrected hyperopia, occupational demands, female gender, systemic diseases such as diabetic mellitus and multiple sclerosis overuse of drugs and geographic factors (Patel., et al. 2006).

In Pakistan the prevalence of Presbyopia is $57.5 \%$ out of which $60.45 \%$ are males and $55.23 \%$ are females. The most common cause of presbyopia is poor affordability and unawareness. Cataract was also a major factor of poor near vision in presbyopes which sustained even after the refractive correction. There was $1.96 \%$ prevalence of blindness in this community. Plus lenses are used to reduce range of accommodation in presbyopes, which brings the near point of accommodation to such a distance which is comfortable for near tasks. Monofocals, bifocals or progressive glasses can be prescribed to the patients with presbyopia. Contact lenses can also be a treatment option for presbyopes.

While discussing the mechanism of accommodation it was noticed that the increased refractivity of the eye was probably brought about by a change in the balance between the elasticity of the matrix of the lens and that of its capsule, allowing it to assume a more globular shape. As age advances, several factors combine to diminish the accommodative power. The lens becomes harder and less easily moulds because the elastic force of the capsule is no longer greater than the resistance of lens substance. The lens therefore tends to set in an unaccommodated form. Moreover, the progressive increase in the size of the lens together with similar changes in the cilliary body reduce the circumlental space so that the zonule becomes slackened, reducing the accommodative ability. Although there is evidence that some weakening of the ciliary muscle occurs as age advances, especially in the later years of life. As a result it becomes more and more difficult to see near objects distinctly. This loss of accommodation is not to be considered as abnormal, and it proceeds gradually throughout the life without any sudden alterations. At first no inconvenience is experienced, but eventually a time comes when the near point has receded beyond the distance at which the individual is accustomed to read or to work or beyond the distance at which his arms allow him to hold the printed page, and the being unable to see clearly. It becomes seriously inconvenienced for him to perform near tasks, a condition known as presbyopia (Duke E.S., 1978).

Anyone at the age of 35 or over 35 is at risk for developing presbyopia. Everyone experiences some loss of focusing power at this age but in some patients it is more noticeable. Some of symptoms and signs of presbyopia include hard time reading small points, eye stress and strain, and difficulty to see close objects, headache, and increase working distance. Presbyopia can be diagnosed by comprehensive dilated eye exam. Exams are mostly recommended after the age of 40 to detect presbyopia.

Presbyopia" is a Greek word that mean "old eye". It is a condition in which eye gradually loses the ability to see things clearly. It is an aging process which mostly starts after the age of 40.IT has been observed that patient can see the things clearly when material is held farther away from his/her eyes. A natural crystalline lens is present inside the eye behind coloured Iris. When we are young, our lens is flexible and soft, so it can easily change its shape. This let us focus on objects both far away and close-up. But as we are growing old (most probable age is 40 ), the lens becomes more rigid and it cannot change its shape easily. So it becomes harder to read, thread a needle, or do other close up tasks. As aging process is irreversible and aging is main cause of presbyopia, so there is no way to stop or reverse the normal aging process. The only thing we can do is to correct presbyopia with near eye glasses or contact lenses. We can also go for surgical interventions (Boyd. K., et al. 2007).

Presbyopia is a physiological condition that causes general reduction in the amplitude of accommodation and it is a growing cause of visual disabilities due to aging demographic of the global population. Accommodation has considerable interaction with vergence response; pupil response and binocularity. Presbyopia affects people from the 4th decade of life and is characterized by accommodative loss that leads to negative effects on vision-targeted health related quality of life (Andualem H.B., et al. 2003).

Presbyopia is a condition in which natural crystalline lens changes its shape, which makes it difficult to focus on close objects. It may seem to occur suddenly, but actually it is a phenomenon in which sight reduction occurs over a number of years. Presbyopia mostly becomes noticeable in the early to mid 40's.It is not considered a disease because it is a normal aging process which cannot be prevented. Some signs of presbyopia include blurred vision at normal working distance such as arm's length, holding material away from the eyes, eye ache along with headache when doing close tasks. A comprehensive optometric examination will include testing for presbyopia. Presbyopia can complicate other common vision conditions like near sightedness, far sightedness and astigmatism. The effects of presbyopia will continue over lifetime. So, presbyopic patient periodically needs to change eye wear to maintain clear and comfortable vision. It is actually part of an aging process of the eye and can be easily corrected. Technically, it is the loss of the ability of eye to see objects that are near. It is not a disease and as natural as wrinkles which affects every body at some point in life. It is often confused with far sightedness, but both are different conditions entirely (Michael., et al. 2017).

Presbyopia is a decline in accommodation which diminishes the ability of the eye to focus on near object. It causes near vision impairment and affects near work. Inability to see fine details and blurred vision at the customary near-working distance are hall- 
marks of presbyopia. Presbyopia cannot be cured, but individual can compensate or correct it by wearing single vision, bifocal or progressive eye glasses and recently contact lens can be a good option too. Generally, a convex lens is used to make up for the lost automatic focusing power of the eye. People living with presbyopia suffer with their day-to-day activities due to reduce near vision. Even though presbyopia is early corrected, globally, there are 617 billion (49.7\%) presbyopic patients who had no access to spectacles. Uncorrected presbyopia has significant effect on the quality of life particularly in the life of teachers and computer users (Oduntan 0, 2018).

Presbyopia affects the performance of patients and also creates the burden on economy of any country. There are a number of factors which lead to presbyopia such as a gradual reduction in zonular tension, gradually altered anterior segment geometry, increase in lens thickness, changes in crystalline lens material due to the aging process and the accommodation mechanics by which the ciliary muscles position is altered thus leading to flattening of crystalline lens and decrease in its power. The initiation of presbyopia also depends on number of factors such as refractive errors, working distance, visual requirements, race, gender, geographic factors and environmental elements such as temperature and brightness (illumination) but age is the main risk factor.

Factors which may contribute in the early onset of presbyopia are cardiovascular diseases, systemic diseases such as diabetes mellitus, multiple sclerosis, myasthenia gravis, anemia and influenza and drugs which mostly contribute are antidepressants, antihistamines, antispasmodic, diuretic, anti-anxiety, and chlorpromazine, antipsychotic. External factors such as stress, psychological issues and environmental issues also have a major role in presbyopia onset. Asthenopic symptoms, diplopia, drowsiness and increased working distance are main frequent complaints with presenting complaint of severe headache. As far as the management of presbyopia is concerned, multiple treatment options are available for it. First of all, we have to calculate the accurate near add of the patient. In this regard, amplitude of accommodation is a unique measurement for each eye separately. It is necessary to provide the weakest and most appropriate near add to presbyopic patient (Andualem H.B., et al. 2017).

Patient only uses half of the accommodation and remaining half is reserved. There are a number of methods which are used for the determination of near addition world wide such as increasing plus lens, balance range of accommodation (NRA/PRA),crossed cylinder test, amplitude of accommodation method, age expected addition method, dynamic retinoscopy and near douchrome subjective preference are considerable. Calculation based on the amplitude of accommodation method can provides accurate near addition in a number of presbyopic patients. Age related near addition values have shown a difference compared with the amplitude of accommodation measurement. Some studies favor the age related addition as compared to amplitude of accommodation method (Goss., et al. 2002).
The results of some studies showed that all the methods used to evaluate presbyopic add gave almost similar near addition results. In order to clear the discrepancy between different methods and find a precise technique of determined addition, Andualem conducted a study to establish the level of agreement between the two methods including the amplitude of accommodation and age related addition method. The results showed that age related method could provide accurate near addition in majority of the presbyopic patients (Andualem H.B., et al. 2017).

Presbyopia isn't a disease or error of refraction; it is a physiological condition of eye. Change in refraction occurs after 40 years of age. Refraction change is same in both eyes in presbyopic individual prescription but it can be different in case of anisometropia. Presbyopia exact cause is still idiopathic but there is overwhelming manifest that it occur due sclerosis of lens substance. Hardening is due to chemical changes in lens protein. As lens become harder, converging and accommodative power decreases and person is unable to perform near tasks. Two types of changes occur in presbyopia: lenticular and extra lenticular. In lenticular change elasticity of lens capsule material is reduced and there is increment in disulphide bond between lens and capsule. Whereas in extra lenticular change, cilliary muscle changes occur. When these muscles contract, zonular tension is increased, anterior and posterior zonule relax, central surface of lens become steep and peripheral lens surface flatten that ceases the optical power (Khurana A.K, 2013).

Accommodation is the ability of eye to focus divergent rays that arise from near point on retina. It has two types, physiological accommodation and physical accommodation. First type presents contraction of ciliary muscle and second one represents changes in refractive power of eye. Physiological accommodation can be measured by myodiopters while physical accommodation is calculated by diopters that are obtained by dividing 100 by p.Range of accommodation (a) is distance between punctum proximum (p) and punctum remotum ( $r$ ) this makes $a=r-p$

Punctum proximum is defined as amount of accommodation differ according to age. Punctum remotum is in front of eye in emmetropia and myopia while performing static refraction. In hypermetropia it is behind. Puntum proximum changes with age in children it's about $7 \mathrm{~cm}$, at age of 30 its $14 \mathrm{~cm}$ at the age of 40 it is $30 \mathrm{~cm}$

RAF rule Instrument that asses visual abilities like convergence and accommodation. Measurement of accommodation and convergence is tested by RAF rule( Royal air force rule) its binocular instrument to measure objective and subjective convergence and accommodation in $1 \mathrm{~mm}$ increment. It serve as primary diagnosis of convergence insuffiency that cause asthenopic symptoms and has negative effect on quality of life that results in difficulty of near work. As this instrument introduced it's widely used in world now (Duke-Elder.S, 1998)

If presbyopia is not corrected, the functional vision is likely to be disturbed. There are a number of ways to manage the disabil- 
ity of vision caused due to presbyopia; there is simply no alternative to the accommodative ability of non-presbyopic eye. Presbyopia can be classified into different types. Incipient Presbyopia is also known as borderline, beginning or pre-presbyopia. The main symptom is that person feels difficulty in reading the small prints. It is the earliest stage of presbyopia at which signs and symptoms can be documented. At this stage patients may need reading glasses but he performs well even without glasses and may prefer to remain uncorrected. When patient faces gradual decline in amplitude of accommodation, he eventually reports visual difficulties which confirm the type of presbyopia known as functional presbyopia. There is a critical interaction between amplitude of accommodation and near vision demand of patient. The age at which symptoms of presbyopia appear, varies from person to person. Absolute Presbyopia is a premature type of presbyopia in which the main factors are environment, nutritional deficiencies or use of certain drugs. The accommodative ability of patient is no longer sufficient for the near vision demands of patient at an earlier age than expected. Nocturnal presbyopia is type of presbyopia is results from dim light. Increased pupil size and decreased depth of field in dim light causes this reduction in range of clear vision. The major risk factor in case of presbyopia is age. Trauma is another risk factor which can lead to premature presbyopia. Systemic diseases can also give rise to presbyopia at an earlier age than expected. Certain drugs can also pay role in development of presbyopia. Hyperopia if remained uncorrected requires additional accommodative demand thus leading to presbyopia. There are certain occupations which require extensive near work. Onset is earlier in females as compared to males. Proximity to equator can also be a risk factor (Gary L.M., et al. 2010).

\section{Relaxation theory}

Relaxation theory best describes the accommodative functions. This theory states that constriction of ciliary muscles is responsible for accommodative response, due to which zonules release tension. The suspensory ligaments are inserted at a specific position on equatorial and anterior lens capsule. When zonules are relaxed, they shift the lens contents forward, thus the anterior lens surface becomes more convex (Gary L.M., et al. 2010).

Normally the lens moves forward during growth. This forward movement of lens alters the angle at which zonules are attached at the capsule of lens. If hypertrophy of connective tissues occurs, the normal points at which zonules are attached can be altered. Some other theories suggest that up till seventh decade of life, the contraction force is maintained by ciliary muscles This suggests that changes in ciliary muscles have no effect on amplitude of accommodation and presbyopia only depends on changes in lens. The pharmacological studies show that to produce maximum amount of accommodation at any age, maximum ciliary muscle contraction is needed. The ability of capsule to reshape the lens depends on contraction of ciliary muscles and to be limited by lens substance resistance to change in shape. This resistance (decreased potential to control lens) is mainly due to growth of lens fibers, ciliary muscles or lens capsule [1].
Classical explanation of accommodative function of eye is the relaxation theory. Accommodation response is actually ciliary muscle constriction, which releases tension on the zonules. Because of strategic position of insertion of suspensory ligaments on the equator or anterior of lens capsule, and to elastic properties of capsule, this relaxation of zonular tension shifts the contents of lens forward, and surface of anterior lens become more convex. Actually presbyopia is a condition which can be defined as age-related hardening of lens substances and the lens unable to be molded by the tension exerted by capsular forces. So more studies focuses on the role of lens capsule and ciliary body, diminution of accommodation by increasing age continues to attribute to hardening or sclerosing of the nuclear lens tissue and then reduce elasticity of the lens (Gary L.M., et al. 2010).

The mechanism of normal lens growth, ciliary muscle development, zonular fiber structure and placement, lens capsule properties have also been considered that presbyopia has three causes: normal ciliary muscle growth, normal lens growth, change in the tissue elasticity. The lens diameter increase so create the "Slack" in the suspensory ligament and a loss of ciliary muscle power available for flattening the lens. In normal growth the lens moves forward, altering the angle at which the zonule fiber attaches to the lens capsule (Gary L.M., et al. 2010).

Hypertrophy and the proliferation of connective tissue of the ciliary body alter the point of attachment of the zonules. Speculation that these normal alternations in the anterior part of the eye contribute to presbyopia is supported by studies suggesting that surgically increasing the lenticular space can increase accommodative ability. Studies evaluating the effect of capsule elasticity on lens molding and ciliary muscle contraction force, attributed presbyopia to three factors, changes in lens capsule elasticity, normal lens growth, and increased lens resistance despite increasing ciliary body muscle force. Some research using impedance cyclograph documented that the ciliary muscle maintain contraction force until the seventh decade of life, it then suggest that presbyopia depends on lens change alone and the ciliary muscle contraction is needed to produce maximum accommodation at any age. So in presbyopia the ability of capsule to reshape the lens was found to depend upon ciliary muscle contraction and to be limited by lens substance resistance to change in shape. And the resistance and change (loss of potential to control lens) was attributed to sclerosis or growth of lens fibers, lens capsule, or ciliary muscle. And recent MRI and AScan studies have shown that the lens capsule becomes less elastic with growing age (Gary L.M., et al. 2010).

Scanning electron microscopy studies have postulated that by attachment point variation among sets of zonular fiber (zonular) There is a conflict in studies according to the model stimulation that "extra lenticular" mechanism demonstrating that the effects of presbyopia are attributable to a change in a responsiveness of the lens substance alone. But the principle of accommodative mechanics support the idea that the ciliary muscle and body move forward and inward in such a way that pars plans zonular fibers can exert 
traction directly on the anterior zonular fibers, flattening the lens and decreasing it's refractive power. In some studies that contribute to presbyopia include one addressing the role of the choroid in accommodation. The results of investigating the contractile properties of lens fibers suggest the possibility of a molecular basis for accommodation. However the presbyopia still is most commonly attributed to changes in the overall size and shape of lens. The decrease in accommodation begins early in life in childhood or adolescence. In early age adult years into the fifties the physiologic changes that reduce the eyes accommodative power occur gradually. Typically between the ages of 38 to 43 years, these changes reach the stage at which accommodative loss is sufficient to cause the blurred near vision symptoms of presbyopia. Optometric care can eliminate most, if not all, of the visual disability associated with presbyopia. Though not incapacitating if corrected presbyopia without optical correction results in an ability to perform effortless near tasks at a customary working distance without experiencing visual symptoms. Presbyopia has been described as an irreversible optical failure (Stephen C.M., et al. 2010).

Presbyopia as an age related condition so not considered in the same extent as ocular diseases or other disorder that if not treated have permanent adverse effect on vision or ocular health. Almost at same stage in life everyone experience adverse effect on the vision or ocular health. Almost everyone eventually will experience some disability due to presbyopia. Among the rare exceptions are a few patients with very small pupil who benefit from increased depth of focus some who have little need to see fine details at near, and some with anisometropia who function in a monovision fashion using one eye for distance vision and other for near. Optical correction can successfully remediate presbyopia, no matter when the patient seeks treatment because presbyopia cannot be prevented public education and health promotion enhance the detection of presbyopia. In a study done in 1996, more than half of the patient surveyed did not know the meaning of "presbyopia". These have been a number of reports that show accommodation velocity reduces with ageing. An early finding was provided by Allen, who used a reaction timer to measure the time interval of accommodation responses in subjects 7 to 49 years of age and found response times slower for the older subjects. Continuously recorded lens axial thickness during accommodation (David A., et al. 2011).

Accommodation is the ability of the eye to change its focus from distant to near object and vice versa. This process is achieved by the lens changing its shape. Accommodation is adjustment of the optics of eye for keep the objects in focus on the retina as its distance from the eyes varies. In healthy person, the amplitude of accommodation diminishes gradually and it consistently diminishes up to 55 years. At thus age amplitude of accommodation is essentially considered zero and what is measured in clinical practice is depth of focus. When a patient has insufficient amplitude of accommodation at any stage it suffers from presbyopia. Presbyopia tends to manifest at age of 40 to 45 years. The advance of presbyopia is faster at the time of appearance between the age of 40 and 50 approximately $0.25 \mathrm{D}$ across every 2 years, while the increase is much slower at the age of 50 years, around $0.25 \mathrm{D}$ every eight years. We should consider that the onset of presbyopia occurs at an extremely productive stage of life and economic status of patient as well (Khurana A.K, 2013).

The amplitude of accommodation is the maximum potential increase in optical power that an eye can achieve in adjusting its focus. It refers to a certain range of object distances for which the retinal image is as sharply focused as possible. Amplitude of accommodation is measured during routine eye-examination. The closest that a normal eye can focus is about $10 \mathrm{~cm}$ for a child or young adult. Accommodation then decrease gradually with age, effectively finishing just after age fifty. Negative relative accommodation (NRA) was proposed by Joseph Kearney of Oxford university in 1967 as a measure of maximum ability to relax accommodation while maintaining clear, single binocular vision. Positive relative accommodation (PRA) is a measure of the maximum ability to stimulate accommodation while maintaining clear, single binocular vision (Anonymous).

Range of accommodation is the distance between the farthest point at which an object can see clearly with accommodation fully relaxed and the nearest distance at which an object can be observed with full accommodation measured in meters and centimeters Presbyopia is a decline in accommodation which diminishes the ability of the eye to focus on near object. It causes near vision impairment and affect near work. Inability to see fine details and blurred vision at the customary near-working distance are hallmark of presbyopia (Khurana A.K 2013).

According to Hanlon, Nawakayashra and Shiegzawa an error in reading addition is is one of the most common causes of patient's unhappiness with their new spectacle. Most clinics set one or more of the procedures for routine use depending on personal preferences. We feel it would be more reasonable to use the methods that are close to the final addition of both procedures. Indeed, it would accelerate the entire evaluation process. There are number of methods to measure the near add for presbyopia. But we follow the most appropriate method to determine it. For correction of presbyopia number of treatment available such as near glasses, contact lenses, corneal surgeries and now a days most common is laser treatment for presbyopia. We follow the two methods for measuring the near add. Tentative method for measurement of near adds according to age and measurement of near add from amplitude of accommodation through RAF rule. As accommodation changes throughout the life and it widely vary age to age so Donder's table mostly standardized for age expected addition [2].

The amplitude of accommodation reduces with age. Measurements can range from approximately 10DS at age 20 reducing to 4.5DS at age 40.Values can vary depending on depth of focus, poor general health and medication. Measuring the amplitude of accommodation will give an indication of the patient's accommodation ability and range of clear vision and serve as starting point for determining a near addition (Sandy M., et al. 2010). 
A frequently used method of measuring the near add is measurement of amplitude of accommodation through RAF rule at required working distance for patient. As the half of accommodation is reserved and required working distance also the matter of concern in case of measuring near add with help of RAF rule. This study was designed to compare final addition values with age expected addition and the amplitude of accommodation because no general consensus in the literature concerning the most appropriate method to determine the addition. RAF (Royal Air Force Rule) provides a binocular gauge to measure objective and subjective convergence as well as accommodation in $1 \mathrm{~mm}$ increment. The RAF rule is useful before both diagnosis and treatment. This instrument has four sides that have different measurements or functions.one side feature diopter measurements from 20 to 2 .One side features a ruler from 5 to 50 centimetres.one side that test ages 8 through 48.one side that test for convergence, normal, reduced and defective. This instrument is used to measure the objective and subjective convergence points, examining the accommodation and determining the master eye. The RAF rule used for both diagnosis and treatment (Grosvenor T, 2014).

Few studies were design to investigate the relationship among age, the amplitude of accommodation and near addition power for correcting presbyopia. When an individual changes fixation from a distance to a near fixation target the optical system of the eye has the ability to increase its focusing power. This enables the eye to bring the image of the object to clear focus on the retina. This ability is referred as accommodation and the maximum amount of accommodation an individual is able to exert is known as the amplitude of accommodation. Following the studies of Dander's, Duane and other researchers the amplitude of accommodation has been found to decrease with increasing age. This age related decrease in amplitude of accommodation continues until such a point when the individual is unable to exert sufficient accommodation to bring a bear fixated object to focus. This condition is called presbyopia, thus it is necessary for an addition converging lens either or presbyopic contact lens correction. Number of researchers have reviewed different methods of determining this addition power and have concluded that the additional power increases with increasing age as amplitude of accommodation decrease or decline.

\section{Aims and Objectives}

- To evaluate near add in presbyopes by using addition method.

- To evaluate near add in presbyopes by using RAF rule.

- To compare two different techniques of prescribing near add in presbyopes.

\section{Review of Literature}

Evaluating three different methods of determining addition in presbyopia

A research was conducted by Yazdani $\mathrm{N}$ to compare three different methods to calculate near addition in presbyopic patients. The study contained 81 presbyopic patients of age between 40 to
70 years. Near addition values were calculated by three different methods such as dynamic retinoscopy, amplitude of accommodation, and increasing plus lens. Increasing plus lens method overestimated the values of near addition. The average near addition obtained by amplitude of accommodation, increasing plus lens and dynamic retinoscopy were $1.31,1.77$ and 1.68 accordingly. These results show that increasing plus lens method gives $20 / 20$ vision at near in most of presbyopic patients (63.4\%). The results were approximately the same for all the 3 methods and it gives the comparable values of near additions; however, mean near addition in method of increasing plus lens is higher as compared to other two methods. So it was concluded that in presbyopic patients increasing plus lens method is least time consuming with the range of +0.5DS at the $40 \mathrm{~cm}$ of habitual working distance. In this study, it shows that assessment and management of presbyopia is critical since significant functional deficit occur once the condition left untreated. Over correction and under correction of presbyopia can lead to visual disability and it shows negative effects on the quality of life. Near add according to the subject's favorite in term of image clarity and a comfortable working distance. In this study we investigate three different methods of evaluating near addition. The difference between three methods reveals that there is maximum difference of 0.5 diopter between three methods. Among three methods IPL is more optimal and comfort vision can be achieved through this and amplitude of accommodation give least number of near add. Several factors related to the test characteristics and inter-individual difference such as habits, former prescription and visual requirements. Furthermore, patient of same age group may need different addition depend upon the current refractive error. Reduced accommodation for near vision in hyperopes occur by increased sympathetic inhibitory effects caused by an increased underlying parasympathetic as suggested by results on accommodation. Depending on our study, it is determined that the amplitude of accommodation shows least result as compare to other two methods by using push-up method for measuring the amplitude of accommodation according to which by moving the chart toward the eye the angular size of the eye and amplitude of accommodation increases. In contrast, Rustein., et al. observe that the amplitude of accommodation determined by dynamic retinoscopy is $2.7 \mathrm{D}$ greater than that of push up method Dynamic retinoscopy gives us the objective results of amplitude of accommodation. This result of the current study shows that a fast method is important in presbyopic subjects. IPL method can provide the best vision and patient compliance. In conclusion there is a difference between the increasing plus lens, amplitude of accommodation because AA method provides very low number as compare the dynamic retinoscopy. Increasing plus lens gives us the optimal results and clear view. This study includes 81 patients. The most prevalent complaint was near work difficulty $(46.9 \%)$. The frequency of refractive error recorded as (84\%), $(11 \%)$ and $3.7 \%$ for hyperopia, myopia and emmetropia respectively. The opacities of crystalline lens were detected in $41.6 \%$ of subjects. Near addition obtained by amplitude of accommodation, dynamic retinoscopy and increasing plus lens were 1.31, 1.68 and 1.77 respectively [1]. 


\section{Critical views of presbyopic add determination}

This study was performed by Hanlon to review patients who returned to optometric clinic because they were not satisfied with their reading add. The sample consisted of patients ranging age 40-60years. The presbyopic add which was prescribed to the patients was compared to the four commonly used methods of evaluation of near add. The results indicated that chances of error in age based method were very less as compared to binocular cross cylinder method [2].

\section{Accommodation measurements in a pre-presbyopic and pres-} byopic population

This study was performed in University of Houston with the purpose to identify the most appropriate method for measuring the outcome of accommodative restorative procedures in prepresbyopes and presbyopes. Thirty one subjects participated in this study having a mean age of 43.7 years. Three subjective approaches were used to measure accommodation (monocular) including the push-up test, minus lenses and a focometer while 2 approaches measured with Hartinger refractometer in which pilocarpine and minus lenses were used to stimulate accommodation. The results obtained with push-up method were over estimated. The minus lenses and focometer gave comparable results but with low amplitudes in younger patients and high amplitudes in elder patients as compared to the objective methods. When accommodation was stimulated and measured with Hartinger, comparable results were found. The subjects with light irides showed stronger response with pilocarpine as compared to subjects with light iris. The results obtained by Hartinger method were more realistic as compared to the subjective methods. Accommodative amplitudes measured with various methods. For the research, this study shows that the subject were grouped in 5 years age groups. With considerable variability among the subject following four methods shows the result that higher accommodative amplitude were recorded with the push up method and other three such as minus to blurr, focimeter and Barringer measurements showed almost similar amplitudes. Although accommodation recorded with the push up test appears high relative to the values for the other methods. The 2 subjective methods monocular minus to blur and focimeter require a similar task of the subject. Similar results formed for these two results although difference in image imagination (focimeter constant, minus lenses minification) and blur presentation (focimeter; smooth, minus lens: stepwise) exist. Over-estimation occur if we measure the accommodation through these subjective methods, especially in presbyopic population, because of increase of depth of focus Barringer measurements for subject age 51 to 55 with pilocarpine $6 \%$ stimulation were slightly higher than accommodative response measured objectively by Hamasaki and co author in subjects in the same age group. The Barringer was used to make objective measurement of accommodation. The most effective method to objectively determine true accommodative ability was by stimulating accommodation with negative lenses or pilocarpine and measuring the accommodative response with the Barringer coincidence refractometer. One goal of this study was to determine appropriate methods for measuring accommodation to evaluate patient who have accommodative restorative procedure [3]

Loss of visual acuity is the main reason why reading addition increases after the age of sixty

This study was performed in 2001 in University of Bradford by MacMillan, et al. The purpose of this study was to find out that why the near add increases after the age of 55 years even when the accommodative value is zero. Forty four subjects were selected who were aged less than 60 years. Distant and near visual acuity, near working distance, reading add, arm's length and pupil diameter of all the subjects were measured. Three techniques were used for finding out near add including least plus add by $\mathrm{N}$-notation and MN-READ text and cross cylinder method. $2.75+$ /- 0.4D was the mean working distance. The reading add which was found by using $\mathrm{N}$-notation text $(+2.21+/-0.38 \mathrm{D})$ method showed lower values as compared to cross cylinder technique $(+2.53+/-0.44 \mathrm{D})$ or MNREAD text(+2.48+/- 0.49D). It was concluded that decreased visual acuity after the age of 60 years caused a decrease in working distance which in turn is responsible for increase in text angular sub tense. Therefore, greater addition is needed in case when working distance is reduced. The increases in depth of field in case of lower acuity and supra threshold text is responsible for the reading add being less than the working distance [4].

Presbyopia and the optical changes in the human crystalline lens with age

Glasser A and Campbell MC performed a research in which the crystalline lenses from 27 human eyes ranging age 10-87 years were taken. The purpose for this study was to find out the changes in crystalline lens of eye due to age and accomadation. The lenses were applied force through ciliary body and zonular fibers and in the meantime the spherical aberration and focal length of lenses were measured using a scanner laser technique. With increasing age, the focal length of un stretched lenses also increased linearly. The lenses of younger subjects showed changes in focal length due to stretching while lenses of older subjects showed no change. The results showed that the human lens shows optical changes with increase in age. The study also concluded that presbyopic lenses vary in optical properties than those of younger lenses which can accommodate properly. According to this study younger lenses were able to undergo significant changes in focal length with stretching but the lenses older than 60 years of age showed no change in focal length with stretching. This study provides the data about the theory of presbyopia. Further this study provide an evidence that there is substantial optical changes in the lens of human eye with increasing age and during accommodation, since both the sign of spherical aberration and magnitude changes with age and can also change with stretching of the lens. This research also shows that the properties of lens such as optical properties of the older presbyopic lens are different from the younger one According to theory of Helmholtz (1909) theory of accommodation when the eye focused on distance than the ciliary muscle is relaxed. The ciliary 
body diameter reduced and ciliary muscle releases the resting zonular tension The elastic recovery which cause an increase in lens power to enable near objects to be focused on the retina. Presbyopia (the age related loss of accommodative amplitude),has been related to multiple changes that occur in the eye. The age related loss of accommodation was attributed to decrease zonular tension resulting from continued growth of lens. Weale provided no experimental evidence to support his claim that lenticular sclerosis doesn't occur and his contention is not consistent with subsequent experimental findings. Fisher showed that the elasticity of the human lens capsule decreases with aging and the human lenses subjected to rotational forces undergo and age dependent decline in ability to be deformed. Our data from human lens shows that the age dependent ability of the human lens to undergo changes in optical power falls within the range of accommodative amplitude and that beyond about age 60 years, the human lens can no longer undergo changes in optical power with stretching. The age dependent ability of the lenses to undergo changes in focal length can also be seen by looking focal changes in the individual lenses. Brown has shown through scheimpfleg measurements that in the unaccommodated emmetropic eye there is an increase lens curvature with age, without other compensatory change. The term lens paradox has been used to describe the fact that the distance refractive state of the eye is maintain with age inspire of the reported increasing lens curvatures (Lorentz and Handelman 1988). These studies states that the lens power and refractive state are maintained constant with increasing age. Many current theories of the development of presbyopia are inconsistent with experimental data from Fisher. Development of presbyopia and to identity these age-related changes in the eye that shows the loss of accommodative ability with increasing age (Glasser A \& Campbell M.C, 1998).

An evaluation of estimation methods for determining addition in presbyopes

Bittencourt performed a research in 2013 with the aim to compare methods which are used in addition to near vision with that of final prescription used in presbyopes. Eighty subjects were studied with the age range of 40-60 years. Four different techniques were used to calculate near add including $1 / 3^{\text {rd }}$ accomodative demand with positive lens cross cylinder test with initial myopisation, balanced range of accommodation using minus and plus lenses, 1/2 amplitude of accommodation using minus lenses. The add power was then refined until final prescription was obtained. The results showed that add values of near addition were lower as compared to final addition of ADL and BRA methods. The mean differences of tentative and final addition were low for all tests. It was concluded that all tog these techniques gave almost similar results and the tentative add obtained by these methods was close to final prescription. However it was suggested that near add needs to be adjusted according to the work demand of patient [5].

\section{Comparing methods of determining addition in presbyopes}

This research performed by Beatriz Antona in Camplutense University of Madrid consisted of 69 patients with age 51 years or between 40 to 60 years. The purpose of the study was to compare near addition methods determined by different procedures after providing final prescription.in this study we use plus lenses to compensate in reduction of accommodation with increase in age or in presbyopic age. After addition of lenses near point of accommodation come at comfortable distance. Methods we use in this research were dynamic retinoscopy, near duochrome, balance of negative and positive relative accommodation, binocular fused cross cylinder. Results shows that near addition after performing procedures were relative high than final prescription except in case of binocular fused cross cylinder. The net results show that these differences were small in clinical basis (0.25). In 95\% cases limit of agreement differed in substantially higher than $+/-0.50$. The result shows that there is similar behavior among all methods but age expected method give best results [6].

Comparison of different methods of evaluating near add for presbyopia

This study compares different methods of determining near addition. Presbyopic near addition consider following methods such as plus build up (PBU),fused cross cylinder (FCC), NRA/PRA, by using Donard table on age based and trial frame as well. Then compare all the methods aid also repeatedly. In this study 30 patients are selected. And range of ADD of subjects from +0.75DS to +2.50DS and grouped as low (<+1.50 DS) and high $(>+1.75 \mathrm{DS})$ ADD populations. The methods used to determine the near add are fused cross cylinder (FCC), Plus build up PBU), and NRA/PRA using age related ADD table. The test was randomly performed and subjects have asked to visit once more time so that repeat using the same three techniques. And the subjects also had their near add determined using a trial frame optimization TF methods for comparison Hashemi, 2013). And then analysis of the ADD was conducted with the help of cumulative logit model that contain both of visit first one and later one. Add methods trial frame, Fused cross cylinder, (FCC), Plus build up (PBU), and NRA/PRA using age based table and group (low and high), same analysis was conducted for every group. And the results and analysis of comparing all the three methods shows that there is no significant difference between all methods results $(p<0.0001)$. And the results were taken independent of know difference in groups $(\mathrm{p}<0.0001)$. The following significant difference NRA/PRA was 4.304 times more likely to yield a higher ADD then TF trial frame NRA/PRA was 3.027 times more likely to yield a higher ADD then Fused cross cylinder (FCC), NRA/PRA to show highest ADD. So it is concluded that if we compare all the three method of calculating near addition then there is significant difference of near ADD such as the higher values of ADD in case if we measure through NRA/PRA with Age based ADD table other than FCC fused cross cylinder and PBU plus build up. So it result that measuring ADD through NRA/PRA over correct the presbyopia (Achenbach P, 2009).

Method of measuring amplitude of accommodation with RAF rule for calculating near add

To measure the amplitude of accommodation for calculating near add there are five different methods (push up, pushdown, 
push down to recognition, minus lens and dynamic retinoscopy). From five method of determining amplitude of accommodation four are subjective and one is objective. The objective one is dynamic retinoscopy. According to researches objective measuring of amplitude of accommodation is not clinically used in practice, it can use for open view auto refractor and are pupil size dependent (Winn., et al. 1998). According to research of literature, it shows no systemic survey of current routine practice for the measurement of amplitude of accommodation. In standardized patient's research of pre-presbyopic only 36 of 100 optometrist measured amplitude of accommodation some with push up, some with push down and comparable and others with combination of techniques (Shah, 2013). With many of us the rule for measuring amplitude is apt to gather dust in some corner of test room(Coates 1995). Measurement of amplitude of accommodation (AOA) is recommended for routine clinical eye examination in the UK(college of optometrist 2012) for detection of presbyopia. Push up method is ubiquitous (Somers and ford 1983). It is commonest and simple technique to measure amplitude of accommodation (Atchison., et al. 2012). According to the researcher in this method the patient is optically corrected for distant vision, views a detailed test objects and reports when there is the first slight, sustained blur (Rosenfield 2009), and the object place at the near point if the eye and then it's distance from the eye is measured in meters and then converted into diopters. This method is mostly perform through an instrument know as RAF ruler and is well established in clinical practice. Another method for measuring the amplitude of accommodation for presbyopia with the help of RAF ruler is push down method, this method can be measured as a variation on the pushup method in its initial description (Turner 1958), The test is perform by moving the object away from the eye and ask the patient for first clear image. Rosenfield (2009) and Barratt (2013) averaging it's result with push- up method. Push down to recognition is similar to that of push down except that the end point is when the patient first recognizes a target as it moves away from the eye. It termed as the modified push up method. But this method is not used in clinical practice widely. This method is now currently used in some set up (Barratt, 2013) and used in research.

\section{Methods to calculate amplitude of accomodation}

Another research was performed to measure methods of measuring the amplitude of accommodation by using minus lens monocularly. Minus lens is a negative spherical lens that are added to the distance correction until patient cannot maintain the initial accuracy. This test only performed monocularly, because it is a result of excess of accommodation which would disrupt binocularity. Dynamic retinoscopy is also a method to calculate amplitude of accommodation. It require skills by the practitioner (Leon., et al. 2012, Roch., et al. 2007). Overall reports according to this study contain that retinoscopy and minus lens assist repeatable but very less common in clinical practice. So community optometrist measure amplitude of accommodation with normal values derived from population survey. The need of such test in older patient is increased. Several methods are available for measure amplitude of accommodation, the strength and weakness of each test is dis- cussed in this study so it approaches the most common method used in clinical practice is push up method with RAF rule. Amplitude of accommodation is a fundamental optometric procedure for calculating near ADD in presbyopic patients. Some suggestions have made to improve current clinical method of measuring amplitude of accommodation [7].

\section{The presbyopic ADD: Age related trend and a gender difference}

In this study a clinical representation of data shows that the earlier results in describing the time course of presbyopia, as indicated by the magnitude of binocular spectacle add in normal sighted males and females. Initially a steep increase in add requirement beginning in early forties becomes relative slower but still significance beyond the mid-fifties [8]. This study is correlated with the age-related progressive decline in the amplitude of accommodation itself a consequence of physiological changes in the crystalline lens The continuing need for an increase in the positive near supplement beyond the mid to late fifties by which time little or no useful accommodation is available is now attributed to the visually disruptive effects within the high spatial frequency domain but consistent gender differences is revealed in presbyopic corrections. Females are on the greater risk and has greater magnitude then their age matched males gender (Pointer J.S, 2001)

\section{The presbyopic ADD: Influence of distance refractive type}

Distance refractive error such as hyperopia, myopia influencing the rate and magnitude of presbyopia doesn't seem to receive systemic consideration. To address this a series of data on the progression of presbyopia add is obtained. Do this data shows that the influence of distance refractive type on the presbyopia such as myopes shows a curvilinear path over the four decades between 41-80 years of the age, hyperopes shows a two phase rise in presbyopic Add and emmetropia shows a linear pathway in graphical presentation. All the three types of distant refractive errors shows that as age advancing the presbyopic add goes on increasing (Pointer J.S, 2001)

\section{The presbyopic ADD: Magnitude and distribution in a} historical context

Presbyopic add is physiological disorder as age related loss of accommodation and recession of the near point. It is firstly counter the patient in middle age. It is a (binocular) spherical positive power supplement superimposed on any distance refractive correction to encounter near vision difficulties. It shows that the age increasing such as individual in mid-fifties there is deceleration in add requirement beyond this. The linear ascending trend in near lens power versus increasing age advocate since the seventeenth century is at odd with twentieth century clinical observation. It shows this difference is reconciled in body qualitative term if not in magnitude. When typical clinical near add data are superimposed on the accepted distance hypermetric refractive base recorded from early middle age onwards. This result shows that the contest of the age markings found on certain eighteenth century antique near vision glasses and the recommendations attached to the selfselection of reading glasses. 
To evaluate the impact of presbyopic remedy and its effect on nature of vision

The utilization of visual redresses by 529 consecutive patients (matured 36 years to 85 years, $50.4 \%$ female) going to 4 optometric practices in various territories crosswise over London were studied by meeting and finished the nature of vision poll to assess visual side effects. Over portion of the populace (54.7\%) oversaw without glasses probably a portion of the time, while somewhere in the range of 30 and $40 \%$ wore separation, perusing and dynamic exhibitions with those utilizing Progressive Addition Lenses wearing them over $80 \%$ of the time, while those wearing perusing scenes just roughly $25 \%$ of the time. Age, sex and driving recurrence had no impact of $(p>0.05)$, though the separation of the assignment altogether affected $(\mathrm{p}<0.01)$. In all measurements, paying little heed to the far, middle of the road or close haze evaluation, was appraised higher by patients whose fundamental errands were far centered $(n=231,43.9 \%)$, than the individuals who chiefly direct halfway undertakings ( $\mathrm{n}=165,31.4 \%$ ) more regrettable still for those whose principle assignments were close $(n=130,24.7 \%)$, paying little respect to the type of amendment. Dominant part of errands are out there and these had a higher than middle assignments with close centered undertakings being far more detestable. It is critical to talk about with patients the key separation of the assignments they by and large perform and the types of presbyopia rectification utilized from the start (Ahmed Siverdeen, 2018).

Comparison of presbyopic additions determined by the fused cross-cylinder method using alternative target background colours

Forty one presbyopia subjects matured somewhere in the range of 42 and 60 years were tried. Speculative increments were resolved utilizing a red-green foundation letter diagram, and 4 crossframework graphs (with white, red, green, or red-green foundations) which were utilized with the melded cross barrel (FCC) strategy. The last expansion for a $40 \mathrm{~cm}$ working separation was resolved for each subject by abstractly modifying the conditional additions. Here were noteworthy contrasts in the speculative increases acquired utilizing the 5 techniques (rehashed measures ANOVA, $\mathrm{p}<0.001$ ). The mean contrasts between the provisional and last increases were $<0.10 \mathrm{D}$ and were not clinically important, except for the red-green letter test, and the red foundation in the FCC technique. There were no critical contrasts between the provisional and last augmentations for the green foundation in the FCC technique $(p>0.05)$. The interims of the $95 \%$ furthest reaches of understanding were under $\pm 0.50 \mathrm{D}$, and the tightest interim $( \pm 0.26$ D) was for the red-green foundation (Wee S.H., et al. 2010).

\section{Population-based study of presbyopia in Shahro}

Presbyopia was characterized as the remedy of close vision to $\log$ MAR 1 (N8 point) with no less than $1 \mathrm{D}$ of includes power. Mean include control in the age gatherings of 40-44, 45-49, 50-54, 55-59 and 60-64 years was $0.65,1.30,1.70,1.87$ and $2.08 \mathrm{D}$, separately. For every 5-year increment in age, a $0.35 \mathrm{D}$ increment in include control was noted. The predominance of presbyopia was $58.15 \%$
(95\% certainty interim: 56.46-59.84). Presbyopia was progressively pervasive in ladies $(\mathrm{P}<0.001)$ and expanded with maturing more in ladies than in men $(\mathrm{P}<0.001)$. Besides, in the 60-64-year-seniority gathering, $11 \%$ of men and $23 \%$ of ladies were not presbyopic. Compared with different reports, the include control in various age bunches was $0.5 \mathrm{D}$ less, and presbyopia was less predominant. Over half of the more than 45-year-elderly people were presbyopic and $17 \%$ of the more than 60 people were free of this condition [9].

\section{Methodology}

\section{Study design}

A cross sectional study was conducted in a time period from $7^{\text {th }}$ January 2019 till $28^{\text {th }}$ April 2019 in the department of ophthalmology in Madina teaching hospital Faisalabad.

\section{Sampling technique}

All patients included in the study were selected through nonprobability convenient sampling technique. All patients included in this study had problem with near tasks and their age was above 40 years.

\section{Sample size}

The research was carried out with 40 subjects with mean age 61.0 (range 40-70 years). Both male and female patients were included.

\section{Inclusion criteria}

The inclusion criteria of the study were patients of age above 40 years having difficulty in reading or performing near tasks. All the patients had blurred near vision.

\section{Exclusion criteria}

Patients with age of less than 40 years and greater than 70 years were excluded. All cases with media opacity, allergies and infections were excluded. Patients with history of any ocular surgery, ocular trauma, cataract, and ocular pathologies (retinal and optic nerve diseases and ocular muscle palsies) were excluded. Patients with history of glaucoma were also excluded.

\section{Instruments used}

Instruments used in this study include

- RAF rule

- Trial lenses

- Near vision chart

\section{RAF rule}

Royal Air Force Rule is a binocular instrument to measure Accommodation and subjective as well as objective Convergence. It consists of a rule having a length of $50 \mathrm{~cm}$ and a rotating cube having four sides. Each side of the cube has a different target. There is a vertical line with a central dot in the first side. The other three sides have near reading lines.It has a cheek rest which is put on patient's cheek to measure the reading correctly. This ruler can be used for diagnosis as well as treatment. 
The four sides have different functions which are as follows

- $\quad$ One side shows diopter measurements from 20 to 2

- $\quad$ One side has measurements from 5 to $50 \mathrm{~cm}$.

- $\quad$ One side shows ages from 8 to 48 .

- $\quad$ One side tests for convergence if it is normal, reduced or defective.

\section{Near vision chart}

This chart has lines with standard spacing. The near vision is recorded as N5, N6, N8, N10, N12.

\section{Trial lenses}

Convex lenses are used for correction of presbyopia. It is also known as converging lens and it is thicker at the center and thinner at edges. The main purpose of using plus lens is that it converges the rays of light and brings them into focus so that they can fall on retina.

\section{Procedure for data collection}

Informed consent of subjects was taken prior to data collection. Two different techniques were applied on each patient to calculate the near addition including age expected addition method and amplitude of accommodation by RAF rule based method.

\section{Age expected addition method}

- First of all, we put the distance refractive correction of patient into the trial frame

- The room's light was on and reading lamp was used.

- Patient was asked about his near tasks and the working distance at which these tasks would be performed was determined.

- The patient was told about the procedure and was asked to cooperate.

- Patient was asked to hold the near vison chart at his comfortable reading distance and we measured that distance with the help of a ruler.

- By using near addition table, we determined a tentative near add for the patient.

- If the working distance of patient was nearer to $33 \mathrm{~cm}$, we added +0.5D to the tentative add of patient.0.50D was subtracted from the tentative add if the working distance was nearer to $50 \mathrm{~cm}$.

- After that we put this addition into the trial frame. Asked the patient to read the smallest size print which was possible. Visual acuity of both eyes was measured.

- We added -0.25D to this addition and asked patient if the letters appeared more clear or blurred. If the visual acuity of patient showed any improvement, we continued adding $-0.25 \mathrm{D}$ otherwise if the vision deteriorated, we did not add it.

- The final near addition and visual acuity obtained by this addition was recorded.
Amplitude of accommodation-based method:

- Amplitude of accommodation is unique for each eye, so it was important to provide addition to each eye separately.

- The patient's distant vision was introduced in the trial frame and one eye was occluded.

- Whole procedure was instructed to the patient.

- The cheek rest was put on patient's cheeks and he was asked to read the smallest optotype which appeared clear to his eye.

- The four-sided cube was moved towards the eye of patient and he was asked to tell when the optotype appeared blurred.

- This distance was recorded and converted to diopters.

- The same procedure was repeated on the fellow eye.

- The mean amplitude of accommodation (AA) between both eyes was also calculated.

- The working distance of patient was also recorded

- Half of the amplitude of accommodation was reserved in case of working distance greater than $40 \mathrm{~cm}$.

- One third of the accommodation was reserved for a working distance less than $40 \mathrm{~cm}$

The near addition value was calculated by following formulae:

1. W.D-1/2AA(in case of W.D $=40 \mathrm{~cm}$ )

2. W.D-2/3AA(in case of W.D less than $40 \mathrm{~cm}$ )

This near addition was introduced in the trial frame and patient was asked to hold the near chart at his comfortable reading distance.

The final near addition and visual acuity obtained by this addition was recorded.

After performing both the procedures the results were compared. The patient was given a specific near vision task and the more appropriate near add was determined. Patient was asked which lenses are more comfortable for him out of the two pairs.

\section{Results}

Total 40 patients of age 40 to 70 were included in our study. The study was conducted in Madinah Teaching Hospital Faisalabad. The duration of this study was almost 2 months According to inclusion criteria of this study only those patients were selected who have not any other active eye disease like glaucoma, cataract, trauma hypertensive retinopathy or any other allergic disease.

\section{Percentage of patients according to age}

Table 1 provides us the information of percentage of subjects according to different age group of presbyopic patients. we conducted this study on 40 subjects of range 40 to 70 years in which $5 \%$ of patients lies in age group of 40 to 45 years, 35\% of patients lies in age group of 46 to 50 years, $22.5 \%$ of patients lies in age group of 
51 to 55 years, $15 \%$ of patients lies in age group of 56 to 60 years, $12.5 \%$ of patients lies in age group of 61 to 65 years and $10 \%$ of patients lies in age group of 66 to 70 years. So this table shows the distribution of subjects according to different age groups.

\begin{tabular}{|l|c|c|c|c|}
\hline & Frequency & Percent & Valid Percent & $\begin{array}{c}\text { Cumulative } \\
\text { Percent }\end{array}$ \\
\hline 1 & 2 & 2.5 & 5.0 & 5.0 \\
\hline 2 & 14 & 17.3 & 35.0 & 40.0 \\
\hline 3 & 9 & 11.1 & 22.5 & 62.5 \\
\hline 4 & 6 & 7.4 & 15.0 & 77.5 \\
\hline 5 & 5 & 6.2 & 12.5 & 90.0 \\
\hline 6 & 4 & 4.9 & 10.0 & 100.0 \\
\hline Total & 40 & 49.4 & 100.0 & \\
\hline
\end{tabular}

Table 1: Age (Binned).

Percentage of patients according to gender

Table 2 it provides the information of percentage of subjects according to gender. It shows that $52.5 \%$ of males and $47.5 \%$ of females were selected for this study.

\begin{tabular}{|l|c|c|c|c|}
\hline & Frequency & Percent & Valid Percent & $\begin{array}{c}\text { Cumulative } \\
\text { Percent }\end{array}$ \\
\hline Male & 21 & 25.9 & 52.5 & 52.5 \\
\hline Female & 19 & 23.5 & 47.5 & 100.0 \\
\hline Total & 40 & 49.4 & 100.0 & \\
\hline
\end{tabular}

Table 2: Gender of patient.

Graph 1 it is graphical presentation of table 2 which shows the graphical view of both genders and their percentage selected for this study. It shows greater number of males selected for this study as compared to females. There were $47.5 \%$ females and $52.5 \%$ males as shown in graph 2 .

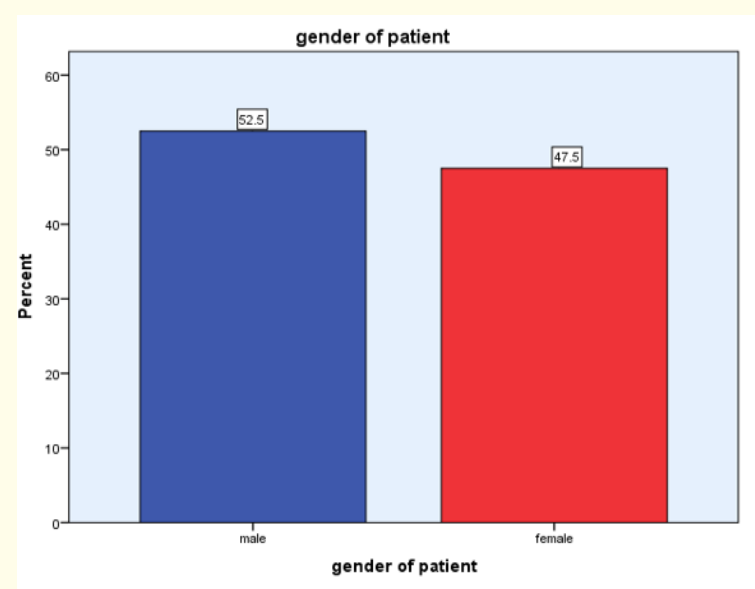

Graph 1

\section{Percentage of patients according to their Professions}

Table 3 this table provides the information of percentage of subjects according to different professions. Total 10 professions are present. It shows that $15 \%$ of patients are workshop owners, $2.5 \%$ are sweepers, $20 \%$ are teachers, $15 \%$ are cooks, $15 \%$ are farmers, $7.5 \%$ are factory workers, $7.5 \%$ are house wives, $7.5 \%$ are watch men, $2.5 \%$ are tailors and $7.5 \%$ are bankers.

\begin{tabular}{|l|c|c|c|c|}
\hline & Frequency & Percent & $\begin{array}{c}\text { Valid } \\
\text { Percent }\end{array}$ & $\begin{array}{c}\text { Cumulative } \\
\text { Percent }\end{array}$ \\
\hline $\begin{array}{l}\text { Workshop } \\
\text { owner }\end{array}$ & 6 & 7.4 & 15.0 & 15.0 \\
\hline Sweeper & 1 & 1.2 & 2.5 & 17.5 \\
\hline Teacher & 8 & 9.9 & 20.0 & 37.5 \\
\hline Cook & 6 & 7.4 & 15.0 & 52.5 \\
\hline Farmer & 6 & 7.4 & 15.0 & 67.5 \\
\hline $\begin{array}{l}\text { Factory } \\
\text { worker }\end{array}$ & 3 & 3.7 & 7.5 & 75.0 \\
\hline House wife & 3 & 3.7 & 7.5 & 82.5 \\
\hline Watch man & 3 & 3.7 & 7.5 & 90.0 \\
\hline Tailor & 1 & 1.2 & 2.5 & 92.5 \\
\hline Banker & 3 & 3.7 & 7.5 & 100.0 \\
\hline Total & 40 & 49.4 & 100.0 & \\
\hline
\end{tabular}

Table 3: Profession.

Graph 2 it shows the graphical presentation of table 3, in which graphical view of percentage of different professions provides the knowledge about the subject's profession. Each peak shows the percentage of each profession separately. The total 10 professions have separate percentage shown in separate peaks in graph like it shows that $15 \%$ of patients are workshop owners, $2.5 \%$ are sweepers, $20 \%$ are teachers, $15 \%$ are cooks, $15 \%$ are farmers, $7.5 \%$ are factory workers, $7.5 \%$ are house wives, $7.5 \%$ are watch men, $2.5 \%$ are tailors and $7.5 \%$ are bankers. All the subjects are distributed separately in their related professions.

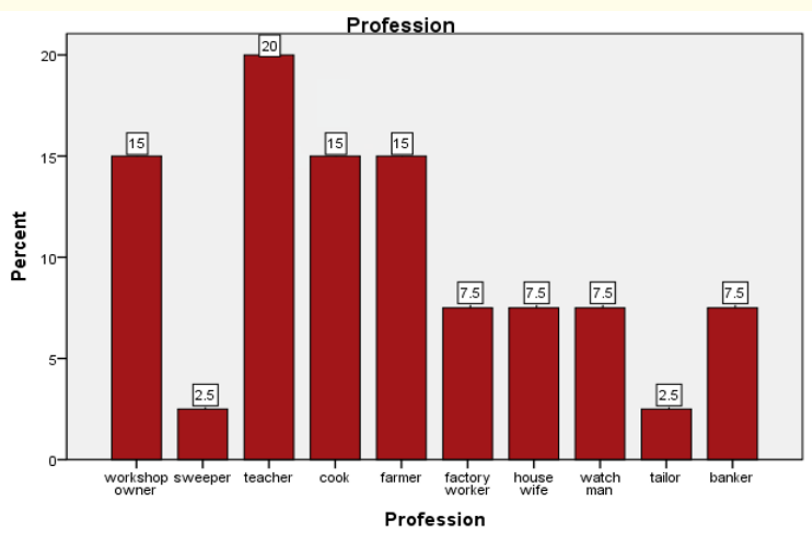

Graph 2

\section{Measurement of Near ADD with age-expected method:}

Graph 3 shows that percentage of subjects according to near add calculated by age-expected method, in which graphical view present the distribution of patient accordingly. Graph shows the percentage of subject as each peak present it separately like 5\% 
of subject has near value of $+0.5 \mathrm{DS}, \%$ has near add range from $+0.5 \mathrm{DS}$ to $+1.00 \mathrm{DS}, 15 \%$ has $+1.00 \mathrm{DS}$ to $+1.50 \mathrm{DS}, 4 \%$ has $+1.5 \mathrm{Ds}$ to $+2.00 \mathrm{Ds}, 6 \%$ has $+2.0 \mathrm{Ds}$ to $+2.5 \mathrm{DS}$. As shown in graph another peak of $6 \%$ has $+2.5 \mathrm{DS}$ to $3.0 \mathrm{DS}$ and remaining $3 \%$ shows the near add value more than +3.00DS

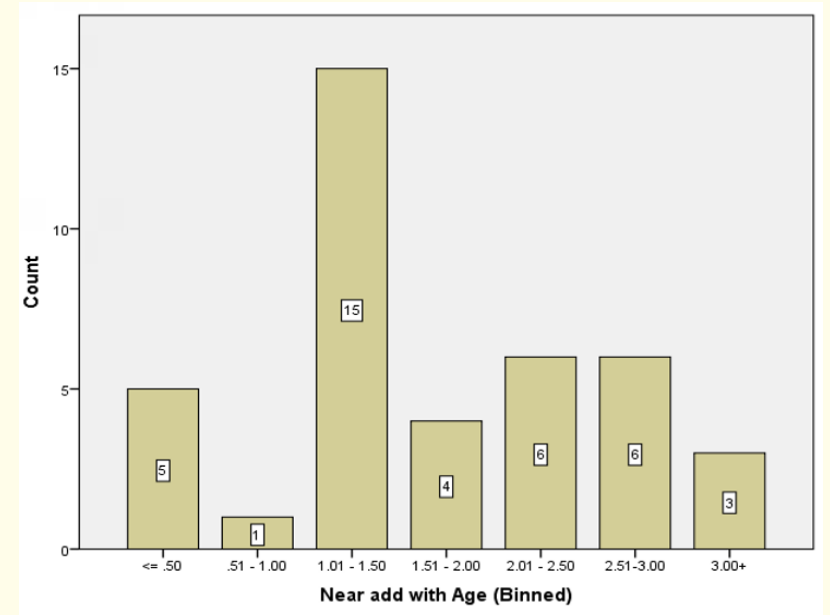

Graph 3

Measurement of near ADD with amplitude of accommodation by using RAF rule

Table 4 provides the information of percentage of subjects according to near add calculated by amplitude of accommodation. It shows the percentage of patients and their distribution accordingly. The table 4 gives us the knowledge as $2.5 \%$ patients near add calculated is $+0.25 \mathrm{Ds}, 20 \%$ has $+0.25 \mathrm{Ds}$ to $0.5 \mathrm{Ds}, 22.5 \%$ has +0.5 Ds to +0.75 Ds, $47.5 \%$ has +0.75 to $+1.00 \mathrm{Ds}, 2.5 \%$ has $+1.00 \mathrm{Ds}$ to $+1.25 \mathrm{Ds}$, other $2.5 \%$ has +1.25 to $+1.5 \mathrm{Ds}$ and remaining $2.5 \%$ patients near add calculated is greater than +1.5 Ds. So this table shows the near add calculated by this method which is very low as compare to near add appreciated by the patients calculated from age-expected method.

\begin{tabular}{|l|c|c|c|c|}
\hline & Frequency & Percent & $\begin{array}{c}\text { Valid } \\
\text { Percent }\end{array}$ & $\begin{array}{c}\text { Cumulative } \\
\text { Percent }\end{array}$ \\
\hline$<=.25$ & 1 & 1.2 & 2.5 & 2.5 \\
\hline $.26-.50$ & 8 & 9.9 & 20.0 & 22.5 \\
\hline $.51-.75$ & 9 & 11.1 & 22.5 & $45 \mathrm{~s} .0$ \\
\hline $.76-1.00$ & 19 & 23.5 & 47.5 & 92.5 \\
\hline $1.01-1.25$ & 1 & 1.2 & 2.5 & 95.0 \\
\hline $1.26-1.50$ & 1 & 1.2 & 2.5 & 97.5 \\
\hline $1.51+$ & 1 & 1.2 & 2.5 & 100.0 \\
\hline Total & 40 & 49.4 & 100.0 & \\
\hline
\end{tabular}

Table 4: Near add with Amplitude of accommodation (Binned).

Graph 4 shows the graphical presentation of table 4 in which graphical view of each group according to near add distributed among is specified by desperate peaks accordingly. As present in graph $5,2.5 \%$ patients near add calculated is $+0.25 \mathrm{DS}, 20 \%$ has +0.25 DS to $0.5 \mathrm{DS}, 22.5 \%$ has $+0.5 \mathrm{DS}$ to $+0.75 \mathrm{DS}, 47.5 \%$ has +0.75DS to +1.00DS, 2.5\% has +1.00DS to +1.25DS, other $2.5 \%$ has +1.25 to $+1.5 \mathrm{DS}$ and remaining $2.5 \%$ patients near add calculated is greater than $+1.5 \mathrm{DS}$. This graph gives us the information of least amount of near add calculated by this method which is not clinically in practice and appreciated by patients as well.

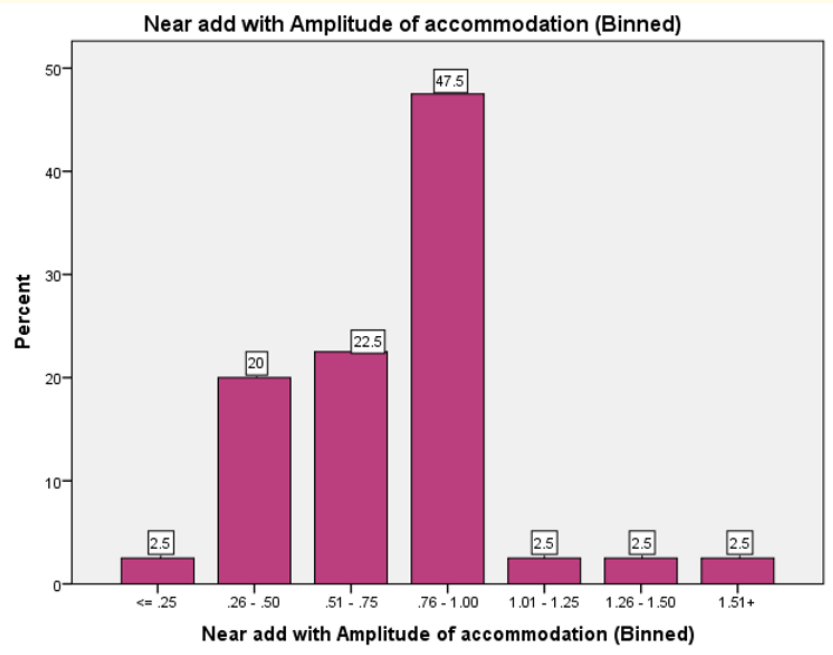

Graph 4

Relationship between age and near add calculated by Amplitude of accommodation with RAF rule

Graph 5 gives us the graphical view two variables which shows the relationship of age and near add calculated by amplitude of accommodation. The main importance of this graph is the separate peaks for each age group and near add calculated accordingly. The first peak shows the near add value of +0.75DS to +1.00DS in age range from 40 to 45 years. Same +1.0DS to+1.25DS of near add calculated in age group of 46 to 50 years, near add greater than $+1.5 \mathrm{DS}$ calculated in the age group of 51 to 55 years, +1.25DS to +1.50DS calculated in age group of 56 to 60 years, $+0.75 \mathrm{DS}$ to $+1.00 \mathrm{DS}$ calculated in age group of 61 to 65 years and same +0.75DS to 1.00DS calculated in age group of 66 to 70 years. This graph 5 shows that near add calculated by amplitude of accommodation gives least amount of near add particularly in higher age groups and the reading add calculated doesn't meet the need of patients. In early onset of presbyopia, the near add calculated by amplitude of accommodation via RAF rule is mostly similar to those of near add calculated by age-expected. And also appreciate by the presbyopes.

Relationship between age and near add calculated by ageexpected method

Graph 6 shows the graphical view of all variables in each peak separately. It presents the relationship between age and near add calculated by age-expected method. Like first peak in graph present the near add range of +1.00DS to +1.5DS in age group of 40 to 45 years, 1.5DS to +2.00DS appreciated by age group of 46 to 50 years, +2.00DS to +2.5Ds appreciated by age group of 51 to 55 years, 


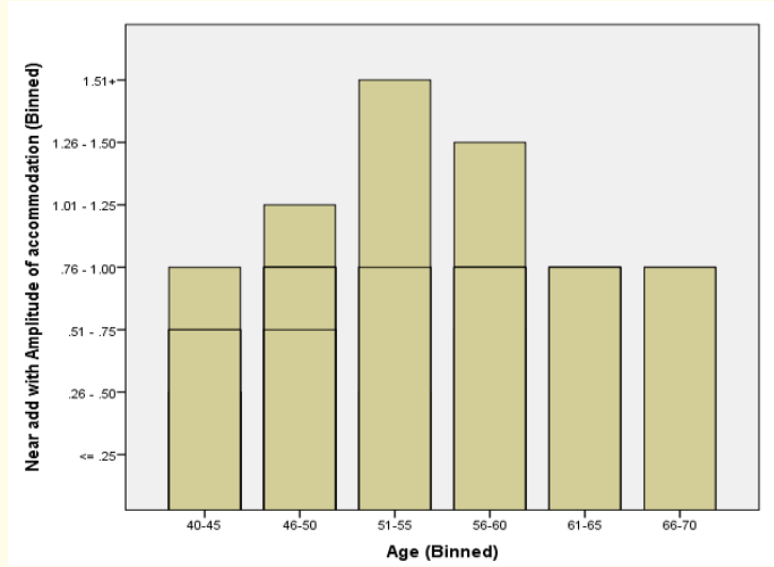

Graph 5
+2.50DS to +3.00DS appreciated by age group of 56 to 60 years. And near add greater than $+3.00 \mathrm{DS}$ appreciated by age group 61 to 66 and same from age group of 66 to 70 years. This graph 6 shows that relationship between age and near add calculated by ageexpected method is actually the appropriate method to calculate near add because majority of patients appreciate this reading add which gives them comfortable vision at near. It is the gold standard method of calculating near add for patients.

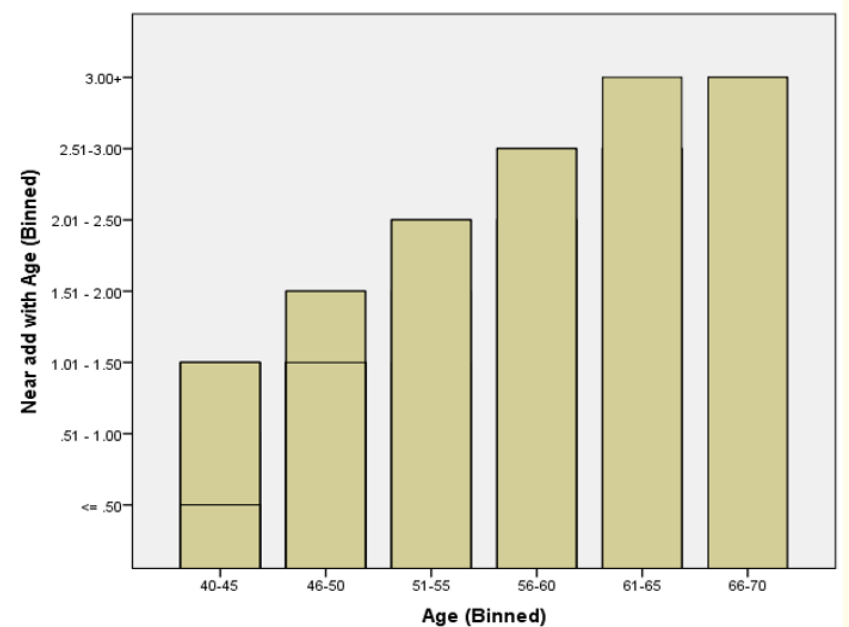

Graph 6

Comparison of two different methods of calculating Near ADD

Table 5 it gives the mean values of two methods, it is the descriptive analysis of two different methods by using data of both methods, which shows the least amount of near addition calculated by amplitude of accommodation and gives the optimal and appropriate near addition calculated by age-expected method.

\begin{tabular}{|l|c|c|c|c|c|c|c|c|}
\hline & \multirow{2}{*}{$\mathbf{N}$} & \multirow{2}{*}{ Mean } & \multirow{2}{*}{ Std. Deviation } & \multirow{2}{*}{ Std. Error } & \multicolumn{2}{|c|}{ 95\% Confidence Interval for Mean } & \multirow{2}{*}{ Minimum } & \multirow{2}{*}{ Maximum } \\
\cline { 1 - 4 } & & & & & Lower Bound & Upper Bound & & \\
\hline NA with RAF & 40 & .8625 & .28840 & .04560 & .7703 & .9547 & .25 & 1.75 \\
\hline NA with Age & 40 & 1.5188 & .73246 & .11581 & 1.2845 & 1.7530 & .50 & 3.00 \\
\hline Total & 80 & 1.1906 & .64416 & .07202 & 1.0473 & 1.3340 & .25 & 3.00 \\
\hline
\end{tabular}

Table 5: Descriptive Data.

Mean value obtained by amplitude of accommodation by RAF rule is 0.86 and mean value obtained by age-expected method is 1.51 . In comparison with mean of addition which is determined by two different methods by using One-way ANNOVA, showed a clinically significant difference $(\mathrm{p}=0.000)$. Our results shows that majority of patients mostly older age are comfortable with near add calculated by age-expected method.

\section{Results}

Table 6 shows the results of one way ANOVA, which gives the significant difference $(p=0.000)$ which means that age expected method is reliable and gives comfortable near vision to presbyopes.

\begin{tabular}{|l|c|c|c|c|c|}
\hline \multicolumn{7}{|c|}{ ANOVA } \\
\hline & $\begin{array}{c}\text { Sum of } \\
\text { Squares }\end{array}$ & df & $\begin{array}{c}\text { Mean } \\
\text { Square }\end{array}$ & F & Sig. \\
\hline Between Groups & 8.613 & 1 & 8.613 & 27.800 & .000 \\
\hline Within Groups & 24.167 & 78 & .310 & & \\
\hline Total & 32.780 & 79 & & & \\
\hline
\end{tabular}

Table 6
Graph 7 shows the mean of near addition calculated by both methods. It shows the least amount of near addition calculated with AA by RAF rule and high values show the addition calculated by age-expected method. So age-expected addition is appreciated by presbyopic patients.

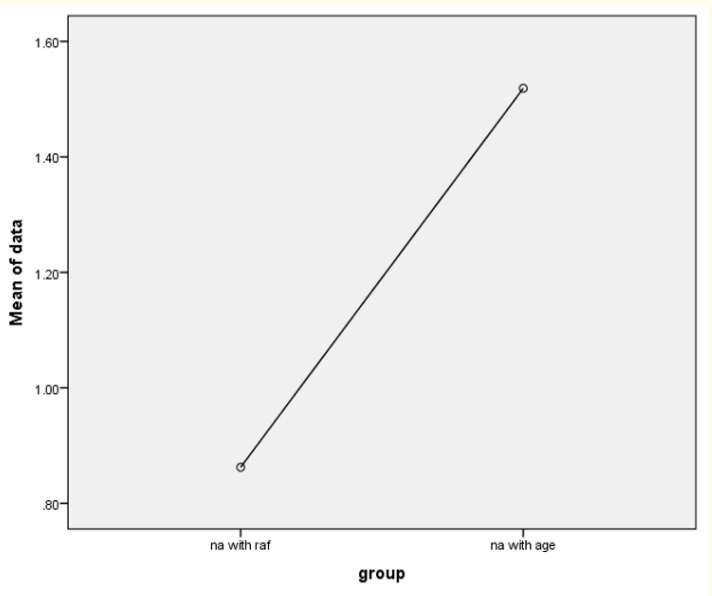

Graph 7 
Graph plotted between NA calculated by AA with RAF rule and age-expected method.

\section{Discussion}

A research was conducted by Yazdani N to compare three different methods to calculate near addition in presbyopic patients. The study contained 81 presbyopic patients of age between 40 to 70 years. Near addition values were calculated by three different methods such as dynamic retinoscopy, amplitude of accommodation, and increasing plus lens. Increasing plus lens method overestimated the values of near addition. The average near addition obtained by amplitude of accommodation, increasing plus lens and dynamic retinoscopy were $1.31,1.77$ and 1.68 accordingly. These results shows that increasing plus lens method gives $20 / 20$ vision at near in most of presbyopic patients (63.4\%). The results were approximately the same for all the 3 methods and it give the comparable values of near additions; however, mean near addition in method of increasing plus lens is higher as compared to other two methods. So it was concluded that in presbyopic patients increasing plus lens method is least time consuming with the range of $+0.5 \mathrm{DS}$ at the $40 \mathrm{~cm}$ of habitual working distance. In this study, it shows that assessment and management of presbyopia is critical since significant functional deficit occur once the condition left untreated. Over correction and under correction of presbyopia can lead to visual disability and it shows negative effects on the quality of life (Yazdani., et al. 2016). Our results showed mean values of 0.86 and 1.51 by AA method and age-based method respectively. Results showed $\mathrm{p}$ value of $0.000(\mathrm{p}=0.000)$. Age expected method gave more accurate results as compared to the AA by RAF rule method. The age-expected add method provided comfortable near vision to majority of patients and they were able to perform their desired near tasks accurately.

Bittencourt performed a research in 2013 with the aim to compare methods which are used in addition to near vision with that of final prescription used in presbyopes. Eighty subjects were studied with the age range of 40-60 years. Four different techniques were used to calculate near add including $1 / 3 \mathrm{rd}$ accomodative demand with positive lens, cross cylinder test with initial myopization, balanced range of accomodation using minus and plus lenses, $1 / 2$ amplitude of accommodation using minus lenses. The add power was then refined until final prescription was obtained. The results showed that add values of near addition were lower as compared to final addition of ADL and BRA methods. The mean difference of tentative and final addition were low for all tests. It was concluded that all these techniques gave almost similar results and the tentative add obtained by these methods was close to final prescription. However it was suggested that near add needs to be adjusted according to the work demand of patient [5]. In our study we used AA by RAF rule and age-based method. The AA by RAF rule method underestimated the near addition values and majority of patients could not perform their near work accurately with the help of this add.We concluded that age-based method is more reliable in majority of the patients as it gave results which were near to our final prescription.
A research performed by Beatriz Antona in Camplutense University of Madrid consisted of 69 patients with average age of 51 years (age range between 40 to 60 years). The purpose of the study was to compare near addition methods determined by different procedures after providing final prescription. In this study we used plus lenses to compensate in reduction of accommodation with increase in age or in presbyopic age. After addition of lenses near point of accommodation comes at a comfortable distance. Methods we used in this research were dynamic retinoscopy, near duochrome, balance of negative and positive relative accommodation, binocular fused cross cylinder, amplitude of accommodation method. Results show that near addition after performing procedures were relative high than final prescription except in case of binocular fused cross cylinder. In $95 \%$ cases limit of agreement differed in substantially higher than $+/-0.50$. The result shows that there is similar behavior among all methods but age expected method give best results [6]. Our study also concluded that age-based method can provide the most accurate results. The near addition calculated by age-expected method showed results which were nearly equal to the final prescription. However, it is recommended that near addition must be adjusted according to the particular needs of patient.

Achenbach conducted a research on presbyopic patients. Thirty presbyopic subjects completed this study. The range of near addition was between $+0.75 \mathrm{D}$ to $+2.50 \mathrm{D}$. The near add was determined using FCC,PBU and NRA/PRA techniques. NRA/PRA was 4.304 times higher than TF and NRA/PRA was 3.191 times higher than PBU. There was significant difference between these methods. Clinician revealed that NRA/PRA method may result in over prescribing of near addition (Achenbach P, 2009). Our study was conducted on 40 subjects and there was a marked difference in results of both techniques in majority of the patients. Age-expected method was the least time consuming method and it provided most accurate results in majority of presbyopes.

We have compared the age-expected method and amplitude of accommodation by RAF rule method. To measure amplitude of accommodation AA in near vision, many presbyopic patient need a plus addition to see the target. This addition has to be considered when calculating the amount of AA and it provides a less accurate value. Several other factors, which relate to the conditions of two tests and the particular characteristics of each subject (visual need, work habits, previous prescription e.t.c) could contribute in the difference of results. In particular, the additions established by the age-expected method reveal that the subjects of similar age may require different additions depending on their degree of ammetropia.

Our results indicate that mean near addition determined by both methods, amplitude of accommodation by RAF rule and ageexpected method are 0.86 and 1.50 respectively. One way ANOVA showed showed significance $(\mathrm{p}=0.000)$. Our results show that ageexpected method in majority of the cases could provide the clear and comfortable vision at near [10-16]. 


\section{Conclusions}

According to our results, amplitude of accommodation provides the least amount of addition and age-expected addition give the optimal and comfortable vision in presbyopic patients. The difference between the near addition calculated by amplitude of accommodation and age expected method is quite significant. Most of the presbyopic patients appreciate the number of reading addition calculated by age-expected method as it provides clear and comfortable near vision.

\section{Recommendations}

- $\quad$ Special seminars should be conducted to educate the public about the age of onset and symptoms of presbyopia.

- $\quad$ A healthy life style should be adopted to avoid the onset of early presbyopia as nutritional deficiency can speed up the onset of presbyopia.

- We used cross-sectional study design to conduct our study as we were given a short time period. There were no follow-ups in our study so we could not determine any post-correction difficulties. To conduct a detailed study, cohort study design would be more appropriate.

- $\quad$ Guide the patient suffering from early onset of presbyopia to change their working distance and hold reading material at a comfortable distance to avoid the excess use of accommodation as in some professions excessive near work is required.

- Recommend pencil push-up therapy to relax the muscles of eye to individuals who perform excessive near work such as computer users.

\section{Limitations}

- We were given a short duration of time, so there were no follow-ups.

- We targeted only on patients coming to Madina teaching hospital Faisalabad.

- $\quad$ Some of the patients were non-cooperative, which complicated our results.

- We conducted our research on a shorter sample size of 40 patients due to shortage of time.

\section{Bibliography}

1. Yazdani N., et al. "Evaluating three different methods of determining addition in presbyopia". Journal of Ophthalmic and Vision Research 11 (2016): 277.

2. Hanlon S., et al. "A critical view of presbyopic add determination". Journal of the American Optometric Association 58 (2002): 468-472.

3. Ostrin LA and Glasser A. "Accommodation measurements in a prepresbyopic and presbyopic population". Journal of Cataract and Refractive Surgery 30 (2004): 1435-1444.
4. MacMillan E S., et al. "Loss of visual acuity is the main reason why reading addition increases after the age of sixty". Optometry and Vision Science 78 (2001): 381-385.

5. Bittencourt, L. C., et al. "An evaluation of estimation methods for determining addition in presbyopes". Arquivosbrasileiros de oftalmologia 76 (2013): 218-20.

6. Antona B., et al. "Comparing methods of determining addition in presbyopes". Clinical and Experimental Optometry 91 (2018): 313-318.

7. Burns DH., et al. "Clinical measurement of amplitude of accommodation: A review". Optometry in Practice 15 (2014): 75-85.

8. Wolffsohn J S., et al. "Accommodative amplitude required for sustained near work". Ophthalmic and Physiological Optics 31 (2011): 480-486.

9. Hashemi H. "Amplitude of accommodation and add power in an adult population of Tehran, Iran". Iranian journal of ophthalmology 25 (2013): 182

10. ADLER P M., et al. "Influence of target type and RAF rule on the measurement of near point of convergence". Ophthalmic and Physiological Optics (2007b).

11. Benzoni J A and Rosenfield M. "Clinical Amplitude of Accommodation in Children between 5 and 10 Years of Age". Optometry and Vision Development (2007).

12. Cleary G., et al. "A randomized intraindividual comparison of the accommodative performance of the bag-in-the-lens intraocular lens in presbyopic eyes". American journal of ophthalmology 150 (2010).

13. Hashemi H., et al. "Amplitude of accommodation in an 11-to 17-year-old Iranian population". Clinical and Experimental Optometry 100 (2017): 162-166.

14. Maheshwari R., et al. "Accommodation: Its relation to refractive errors, amblyopia and biometric parameters". Nepalese Journal of Ophthalmology 3 (2011): 146-150.

15. Marran LF., et al. "Accommodative insufficiency is the primary source of symptoms in children diagnosed with convergence insufficiency". Optometry and Vision Science 83 (2001): 281289.

16. Sterner B., et al. "Accommodation and the relationship to subjective symptoms with near work for young school children". Ophthalmic and Physiological Optics 26 (2006): 148-155.

\section{Volume 3 Issue 10 October 2019 (c) All rights are reserved by Marian Ahmed and Ayesha Mushtaq.}

